\title{
SCOPE OF INTERNET OF THINGS: A SURVEY
}

\author{
UMAMAHESWARI E, AJAY DM, UMANG SINDAL
}

School of Computing Science and Engineering, VIT University, Chennai Campus, Chennai, Tamil Nadu, India. Email: asha.s@vit.ac.in Received: 19 January 2017, Revised and Accepted: 20 February 2017

\begin{abstract}
The advent of internet of things (IoT) has influenced and revolutionized the information systems and computing technologies. A computing concept where physical objects used in daily life, will identify themselves by getting connected to the internet is called IoT. Physical objects embedded with electronic, software, radio-frequency identification, sensors, actuators and smart objects converge with the internet to accumulate and share data in IoT. IoT is expected to bring in drastic changes and solutions to most of the daily problems in the real world. This paper explains in detail the key concepts of IoT, the applications of IoT, the challenges in establishing IoT. This work also explains in detail the future scope of IoT in different fields.
\end{abstract}

Keywords: Computer architecture, Wireless sensor networks, Radio-frequency identification, Security, Radio-frequency identification tags, Internet of Things, Radio-frequency identification technology.

(C) 2017 The Authors. Published by Innovare Academic Sciences Pvt Ltd. This is an open access article under the CC BY license (http://creativecommons. org/licenses/by/4. 0/) DOI: http://dx.doi.org/10.22159/ajpcr.2017.v10s1.19633

\section{INTRODUCTION}

Internet of things (IoT) has become a very prominent in today's era of research. According to Gartner, by 2020 about 20.8 billion connected things will be in use the worldwide [1]. Basic components of IoT are a variety of things - such as radio-frequency identification, near field communication, sensors, actuators, and mobile phones. These things are connected with the daily things possessing distinct address to interact with each other. The different addresses are assigned to all things, to facilitate verification and effective communication. IoT transforms traditional objects into smarter objects by giving them the ability to see, hear, think, perform tasks, and share data. This transformation of things is done by manipulating its underlying technologies. Underlying technologies include embedded devices, pervasive computing, sensor networks, communication technologies, protocols, and applications. The entire structure of IoT is shown in Fig. 1.

Every day activities will become more and more easy and comfortable using IoT. IoT has brought in huge improvements in the domestic sphere, agriculture, smart cities, smart cars, smart homes, etc. In the business sector, IoT has brought in notable advancements in production and service industry. Quality assessment monitoring and improvement have been a major booster to these industries because of IoT. IoT will bring in new opportunities in the information and communication technologies sector. The universal adaption of IoT involves concerns and issues to be answered. Major issues include standardization, scalability, reliability, privacy, and security. This work will explain in detail the concepts, applications, and challenges in IoT.

Section 2 will explain in detail the applications of IoT. Section 3 will explain in detail the challenges in IoT, Section 4 will state the future directions of IoT.

\section{APPLICATIONS OF IOT}

The advent of IoT has brought in massive changes in almost all fields and research areas. The 10 most popular IoT applications are shown in Fig. 2. The following are some of the major fields where IoT is applied:

\section{Automotive and transportation industry}

IoT is tremendously accelerating the speed of innovation in the transportation industry. Connected cars are the latest boom in the

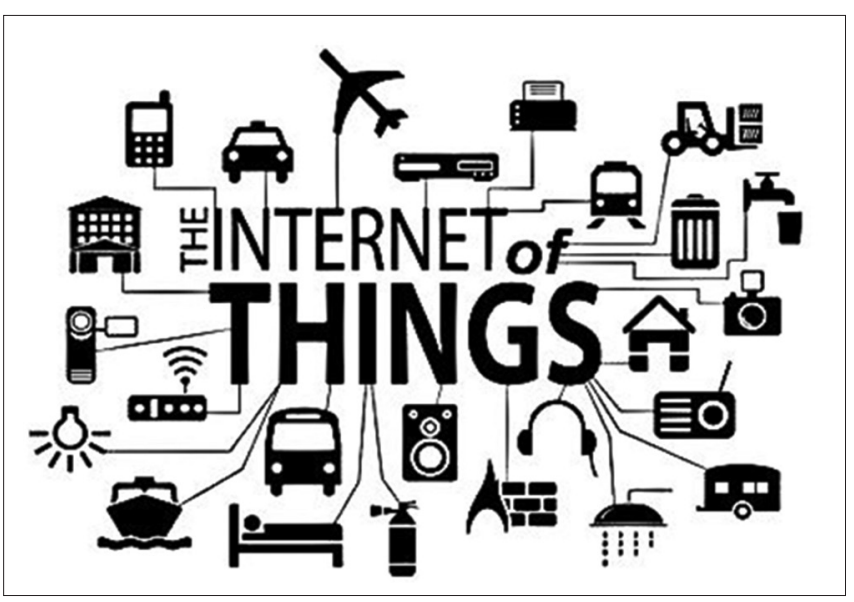

Fig. 1: Internet of things [2]

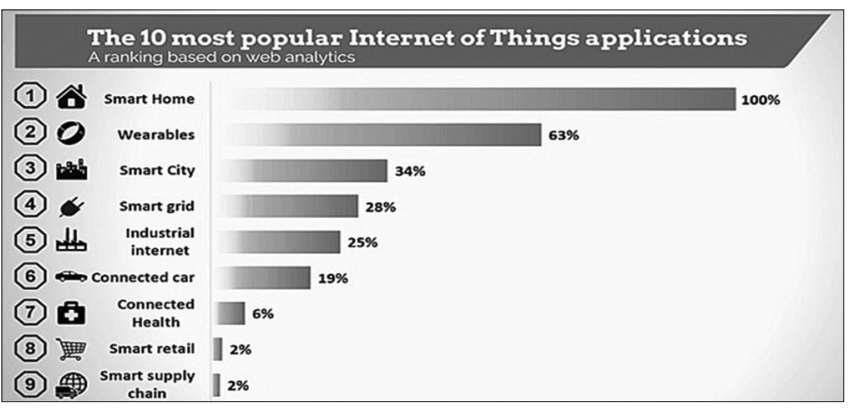

Fig. 2: Popular applications of internet of things [5]

automotive industry. Automobiles are built with numerous connected systems that give the ability for a driver to listen to satellite radio, view and use smart applications, watch streaming videos, check the road navigation, lock and unlock doors with a remote from a distance, inquiry on road assistance and many more. The next generation of automobiles is predicted to be driverless. 
Merging IoT will solve most of the real-time problems in the transportation sector. The more IoT is connected to this sector, the safety of transportation increases. IoT in transportation will help everyone reach their destinations on time safely. The data collected from smart vehicles will help in efficient traffic management, by finding alternate to busy routes on a real-time basis. Roads, where there is vehicle stoppage or accidents, can be avoided by intimating the drivers heading toward that road. Google presently uses GPS location of mobile phones to show the intensity of traffic in Google Maps. Data collected from smart vehicles can be used to trace criminal activities using the smart vehicle data for forensic analysis. Infotainment, wire replacement, telemetry, predictive maintenance, $\mathrm{C} 2 \mathrm{C}$, and $\mathrm{C} 2 \mathrm{I}$ are some of the applications of IoT in these industries $[3,4]$.

\section{Telecommunication industry}

Telecom industry benefits large when connected to IoT. New revenue streams and challenges have emerged by this revolution of connectivity. Tailored consumer applications, wide area IoT solutions, services monitoring and managing can be made available to the users by capitalizing on the large infrastructure, and data provided by IoT. The grade of service (GoS), quality of service (QoS), and quality of experience can be enhanced for the users from the telecom industry. IoT deployment can address the specific GoS and QoS performance levels required. Presently IoT in telecom industry has been giving importance to cost savings. End-to-end solutions for customers, prediction of traffic generation with the data collected are the major uses of IoT/M2M in telecom industry.

\section{Health-care industry}

Health care is one of the biggest revolutionized industries by IoT. Remote monitoring, ambulance telemetry, drug tracking, hospital asset tracking, access control, and predictive maintenance are made available by incorporating IoT in the health-care industry. IoT enables doctors to perform real-time location services by tracking the devices for treating patients. The arrival of patients can be predicted real-time. Medication dispensing devices help in patients not to miss a dose of medicine any time, Niox Mino helps in routine measurements of nitric oxide in patient's breath, UroSense helps in checking the core body temperature of patients, and GPS SmartSole is a shoe-tracking wearable device for patients who forget things [6]. The hand hygiene, i.e., degree of cleanliness in a health-care worker can be detected. Budget can be monitored and with provided the best quality to the patients with minimal expenditure, chronic disease management, detection, and prediction of the outbreak of epidemic diseases, are some of the applications that can be provided by incorporating IoT into health-care industry [7].

\section{Logistics, manufacturing, and supply chain industry}

Logistics and supply chain is one of the industries that have incorporated IoT in large scale. This industry has invested huge capital on IoT implementation and research. IoT plays a major role in logistics and supply chain automation, improved efficiency in maintenance of warehouse, improved quality and cost control in transportation of goods, automated cranes, conveyors, industrial robots, order wrapping, machine labeling, vehicle and order tracking [8]. The primary application of IoT in logistics into manage warehouses by standardizing the processes, controlling the storage and movement of goods by imposing best practices with the IoT data. IoT technology is deployed in targeted areas for tracking behavior of customers, improved situational awareness, and sensor-driven decision analytics. Data collected from IoT have been made the basis for control and automation by converting the collected data into instructions that feedback through the network to actuators that in turn alter processes. Process optimization, optimized consumption of resources, and complex autonomous systems, reduce asset cost, save fuel costs, ensure temperature stability, gain user insight, reduce redundancies, create fleet efficiencies, ensure time in movement, and right quality from the manufacturer are some of the major contribution of IoT in this industry.
The manufacturing industry has been transformed to smart manufacturing industry with the incorporation of IoT in this sector. Production flow optimization based on the customer consumption or customer demand, real-time inventory, asset tracking, employee safety, predictive maintenance, and firmware updates are some of the advancements in this industry after incorporating IoT.

\section{Smart cities}

More than $50 \%$ of world's population lives in the cities and due to this the problems the urban population is on the raise day by day. Problems related to traffic management, sewage management, water supply management, energy management, waste management, etc., need to be addressed in an earlier pace. Incorporating IoT will bring in better solutions for all the above stated urban life problems. The need to move toward smart cities is increasing, and the government has taken steps to implement smart cities $[9,10]$. IoT gives the ability to remotely monitor, manage and control devices. As a result, of innovation in IoT and the offerings it comes with the cities are being transformed by enhancing infrastructure, creating more efficient and cost-effective municipal services, improved public transportation, reducing traffic congestion, and more safety for citizens $[11,12]$. The detailed applications of IoT in smart cities are shown in Fig. 3.

Residential e-meters, smart street lights, pipeline leak detection, waste management, traffic control, surveillance, centralized, and integrated system control.

\section{Wearables}

IoT has brought in immense improvements in the field of wearables and has transformed wearables into health, fitness monitoring devices. Smart watches, smart wrist bands, and smart glasses have been introduced with incorporating IoT, and these wearables act as monitoring devices which help in monitoring blood sugar levels, heartbeat, blood pressure levels, daily fitness activity status, and more. Wearables are used as location and activity trackers. The data collected from wearables are analyzed and used for prediction to improve the living of people.

\section{Agriculture}

Agriculture industry faces immense problems such as increase in water shortage, limited available land for cultivation, and difficulty in managing costs. But increasing population needs increasing in agricultural production for adequate consumption. IoT addresses the above problems by increasing quality, quantity, sustainability, and costeffective agricultural production. IoT helps in remotely monitoring soil temperature, humidity, light intensity, weed growth, soil moisture, crop growth, livestock activity, livestock feed levels, and logistics coordination. IoT provides the ability to remotely control irrigation and harvesting devices. The data collected by IoT sensors can be used for artificial intelligence-based analytics to easily analyze the weather, health of crop and to obtain maximum profit and improve the process of decision-making $[13,14]$.

\section{CHALLENGES IN IOT}

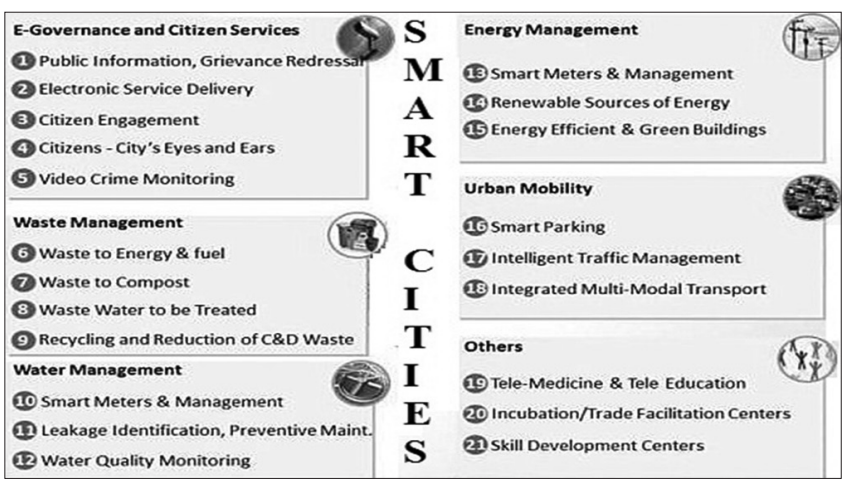

Fig. 3: Internet of things in smart cities [5] 


\section{Sensing a complex environment}

Sensing and collecting data from physical world are not an easy task. Where, how, what data to collect is important to make the process of data analytics more efficient and accurate. The challenges in data collection multiples in a complex environment, where there are more of unwanted data that might be generated. Hence, segregating the unwanted data is a challenging task $[15,16]$. Innovative ways to sense, send, analyze, and deliver information from the complex environment to the storage are needed.

\section{Connectivity}

The development process of IoT devices is easy in a laboratory but deploying an IoT application on a global scale, is a difficult task. Internet is a heterogeneous connectivity of cell towers, proxy servers, firewalls, etc., which disrupt connectivity [17]. Some of the major challenges of IoT connectivity:

- Signaling: Reliable bi-directional signaling is very much essential between connected devices, between devices and server to make sure the complete stream data are transferred to the servers in time.

- Bandwidth: Bandwidth consumption on a cellular network is expensive, and when hundreds of IoT devices send request/response the bandwidth consumption is huge. A light weight network that has the ability to seamlessly transfer data is essential to transfer data between devices and servers.

- Presence detection: IoT devices presence detection over a network provides an exact status of each device every second. This will equip us with the ability to monitor and fix any problems that arise with in the network.

- Standardization: Standardization is the factor that is greatly affecting the growth IoT. IoT will provide cross-platform communication. Too many systems and devices in this world run in their own dedicated platform lacking cross-platform communication. To utilize the potential of IoT to its fullest, a standardization protocol which enables cross communication in essential. A standardized protocol enables easy communication between devices, regardless of the manufacturer with more security $[18,19]$.

\section{Power is critical}

The advent of IoT is increasing the energy demands compared to the demand created by the internet. Data centers powered by the internet require about 30 billion $\mathrm{W}$ of electricity every year. Adding IoT, the electricity requirement is substantially increasing with the advancements of IoT. IoT applications are required to run for years. So the production, utilization and management of power requirements of IoT is a bigger challenge.

\section{Security is vital}

Securing users' data and privacy is one of the biggest concerns of IoT. Detecting and blocking malicious activity is a bigger challenge in IoT.

- Authorization: Proper authorization mechanism is essential while sending and receiving a stream of data from IoT devices and servers.

- Encryption: End-to-end encryption between devices and servers is need to protect the data.

\section{IoT is complex}

IoT application development needs to be easy for all developers, not just to experts. IoT is complex since it has to with stand robust conditions and environments. The application development should make the IoT device reliable. This makes the application development difficult for normal users difficult. Easy development process with open standards is essential for overcoming this issue.

\section{Cloud is important}

IoT data are continuous huge streaming real-time data that should require huge storage and processing speed. IoT data should be processed in a prescribed time, before the data losses its validity. Considering all the above needs cloud infrastructure is very important for IoT [20-22].

\section{Waste disposal}

About 50 million tons of e-waste is produced each year in the United States alone, if IoT spreads throughout the globe then the e-waste produced will become double or triple. Management of e-waste is a huge challenge in incorporation IoT in the various fields.

\section{Storage issues}

Storage of information generated by IoT devices needs huge storage infrastructure and processing speed. Mostly, the data generated by smart devices are needed only for a prescribed less amount of time. However, the problem arises when situation comes when the data needs to be stored for longer period. Efficient policies are essential about what data to be stored for a longer time and what not to store.

\section{FUTURE DIRECTIONS OF IOT}

As discussed earlier, the potential of IoT is huge and it has already drastically changed the face of many fields with its ability to monitor continuously. In the future, IoT is capable of getting incorporated into many more fields. Research has already started in most of the fields on how to incorporate and take advantage of the new technology. The future directions of IoT are expected to take IoT into cloud and to incorporate efficient big data analytics. The trio of IoT, cloud, big data analytics is set to change the technology world. Issues in IoT as mentioned in Section 3 are going to occupy a big part of the research related to IoT. Adding cloud and big data will bring on the issues from those two areas as well. Efficiently tackling all those issues, by increasing more utilization is going to be the future directions in IoT.

\section{CONCLUSION}

IoT is a budding technology, which is rapidly growing and changing the landscape of many areas. The main vision behind IoT is to connect everything and anything at any time, and this is vision has brought in lots of research interests from the world. The dynamic nature of IoT has challenges and concerns to be addressed. This paper explains in detail the basics of IoT, applications of IoT in different fields, challenges in IoT and the future scope of IoT. The motivation for this survey is to bring in a better understanding of IoT and help the research community to bring in more advancements and contributions to the area of IoT.

\section{REFERENCES}

1. Gartner Newsroom. Available from: http://www.gartner.com/ newsroom $/ \mathrm{id} / 3165317$

2. Seo Land. Available from: http://www.seoland.in/how-internet-ofthings-iot-are-going-to-impact-your-business/\#.WDb Bf197IU.

3. Here's What IoT Will Do for Transportation. Available from: http:// www.venturebeat.com/2015/09/19/heres-what-iot-will-do-fortransportation.

4. Application Areas for the Internet of Things. Available from: http:// www.ti.com/ww/en/internet_of_things/iot-applications.html.

5. The Internet of Things Applications Ranking. Available from: https:// www.iot-analytics.com/10-internet-of-things-applications.

6. Applications of IoT in the Healthcare Industry. Available from: https:// www.cabotsolutions.com/2016/02/applications-iot-healthcare-industry.

7. Giusto D, Iera A, Morabito G, Atzori L, editors. The Internet of Things. New York, NY: Springer; 2010.

8. Role of IoT in Logistics \& Supply Chain. Available from: http://www. planetventures.com/role-of-iot-in-logistics-supply-chain.

9. Belissent J. Getting Clever About Smart Cities: New Opportunities Require New Business Models. Cambridge, MA, USA: Forrester Research; 2010

10. Atzori L, Iera A, Morabito G. The internet of things: A survey. Comput Netw 2010;54(16):2787-805.

11. Zhang M, Yu T, Zhai GF. Smart transport system based on the internet of things. Appl Mech Mater2011;48-49:1073-6.

12. Kumar P, Ranganath S, Huang W, Sengupta K. Framework for realtime behaviour interpretation from traffic video. IEEE Trans Intell Transp Syst 2005;6:43-53.

13. Smart Agriculture. Available from: https://www.thingworx.com/ ecosystem/markets/smart-connected-systems/smart-agriculture.

14. Jun-Wei H, Shouyi Y, Leibo L, Zhen Z, Shaojun W. A crop monitoring 
system based on wireless sensor network. Proc Environ Sci 2011; 11:558-65.

15. Challenges in the Internet of Things. Available from: http://www. ti.com/ww/en/internet of things/iot-challenges.html.

16. 5 Challenges of Internet of Things Connectivity. Available from: https://www.pubnub.com/blog/2014-06-17-5-challenges-of-internetof-things-connectivity.

17. The Biggest Problem with the Internet of Things? Available from: http://www.tech.co/biggest-problem-internet-of-things-2015-09M

18. Aggarwal NA, Sheth A. The internet of things: A survey from the datacentric perspective. Managing and Mining Sensor Data. New York: Springer; 2013.
19. Singh AD. Internet of Things: Perspectives, Challenges and Opportunities, International Workshop on Telecommunications (IWT 2013). Brazil: INATEL, May 6-9; 2013.

20. Gubbi J, Buyya R, Marusi S, Palaniswamia M. Internet of Things (IoT): A Vision, Architectural Elements, and Future Directions, Technical Report CLOUDS-TR-2012-2, The University of Melbourne, June29; 2012.

21. Ajay DM, Umamaheswari E. An initiation for testing the security of a cloud service provider. Smart Innovation, Systems, Technologies. Switzerland: Springer Publications; 2016. p. 35-41.

22. Ajay DM, Umamaheswari E. Why, how cloud computing - How not, and cloud security issues. Glob J Pure Appl Math (GJPAM) 2016;12(1):1-8. 